\title{
RESVERATROL REVERSES BRAIN GLUTATHIONE SYSTEM INVOLVED NEURONAL LOSS AFTER IMMOBILIZATION STRESS
}

\section{T. Kalpana ${ }^{1}$, Ramya Sree A *2, B. Naveen Kumar ${ }^{3}$.}

${ }^{1}$ Associate professor, Mamatha Medical College, Khammam, Telangana, India.

${ }^{* 2}$ Assistant Professor, Department of Anatomy, Mamata Medical College, Khammam, Telangana, India.

${ }^{3}$ Associate Professor, Department of Anatomy, Mamata Academy Of Medical Sciences, Bachupally, Hyderabad, Telangana, India.

\section{ABSTRACT}

Background: This study evaluated the antioxidant effects of Resveratrol on stress-induced neuronal loss in rat brain involving brain glutathione system.

Materials and Methods: The control rats received vehicle while another set of rats received 21 days restraint stress. The third and fourth group received similar intensity of restraint stress as well as either $10 \mathrm{or} 20 \mathrm{mg} / \mathrm{kg}$ dose of resveratrol respectively. The cognitive test included passive avoidance test. This was followed by estimation of reduced glutathione and glutathione reductase enzyme levels in brain homogenate and histomorphological study of hippocampus and medial prefrontal cortex.

Results: Restraint stress has resulted in poor retrieval of learning behaviour and resveratrol has enhanced retrieval of learning behaviour in stressed condition in passive avoidance task. Both reduced glutathione and glutathione reductase levels were reduced after restraint stress and resveratrol at both the doses has normalized their levels. Restraint stress has affected CA3, CA2 and dentate regions of the hippocampus and also medial prefrontal cortex. In all these areas resveratrol has minimized neuronal loss which were due to chronic stress.

Conclusion: From the results of the present study we conclude that stress induced oxidative damage involves brain glutathione system and which in turn could be one of the causes for neuronal loss and resveratrol suggests to protect the brain against stress in rat model.

KEY WORDS: Resveratrol, Restraint stress, Reduced glutathione, Glutathione reductase, Hippocampus.

Address for Correspondence: Dr. Ramya Sree A, Assistant Professor, Department of Anatomy, Mamata Medical College, Rotary Nagar,Khammam. Telangana - 507002, India.

Mobile No: 9885658025 E-Mail: ramya.jerus@gmail.com

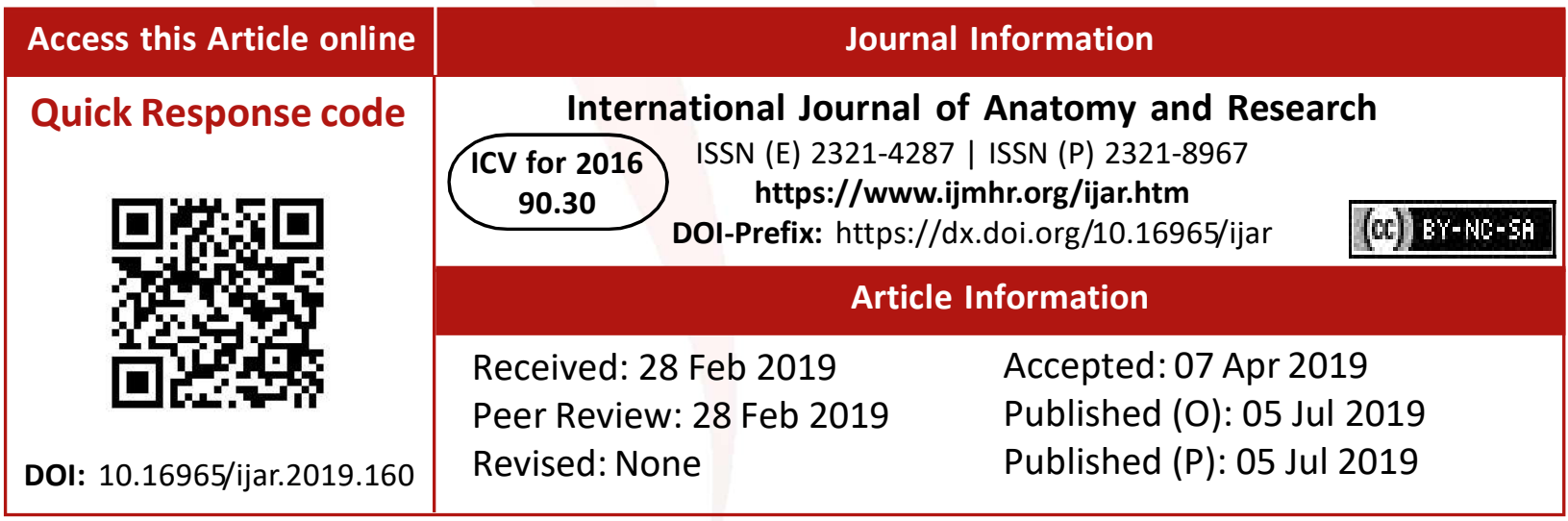

\section{BACKGROUND}

Altered hypothalamo-pituitary-adrenocortical axis causing free radical generation in brain is believed to be the one of major cause for stressinduced neurotoxicity in humans and animals [1]. Stress can amend cytoplasmic constancy by oxidative damage in brain which inturn involves behavioural, biochemical and morphological changes.Glutathione system in brain is one of the major antioxidant defence system to combat various neuronal insults. Reduced glutathione is an intrinsic antioxidant which neutralizes the reactive oxygen species and prevents further cellular damage. Further assay of glutathione 
reductase enzymeprovides information about extent of oxidative stress in terms of formation of reduced glutathione intracellularly.

Resveratrol is a phytoalexin produced by several plants that is sold as a nutritional supplement. Neuroprotective properties of resveratrol are exerted by modulating numerous detoxifying enzymes [2]. It is known to exert its neuroprotective effects through free radical scavenging activity in ischemic conditions [3]. There is a wide ranging list of clinical trials being conducted all over the world to test resveratrol in patient with, Alzheimer's disease (AD), and aging (obesity, metabolic syndrome \& diabetes). It is also stated to be effective against neuron cell death and cell dysfunction [4].

Keeping these results in mind, there is a basis to consider that resveratrol by its antioxidant or other undefined mechanism could reverse the level of neurotoxic effects at behavioural, biochemical and cellular levels against stress. This study would provide a therapeutic strategy to combat the stress-induced neurotoxic effects.

\section{MATERIALS AND METHODS}

Animals and housing conditions: In-house bred male Wistar rats (weighing $220 \mathrm{~g} \pm 20$ ) were selected for the study. The rats were maintained under controlled conditions of light ( $12 \mathrm{~h}$ light and $12 \mathrm{~h}$ dark cycle), temperature $\left(22 \pm 3^{\circ} \mathrm{C}\right)$, and humidity (approximately $50 \pm 10 \%$ ). Allrats were maintained on the standard rat food and water ad libitum. For housing the rat plastic cages with paddy husk as beddingmaterial was used. The institutional animal ethical committee hasapproved this research protocol.

\section{Animal groups $(n=6)$}

Group 1 (Control): Control and received sodium carboxymethylcellulose as vehicle

Group 2 (Stress): Received 21 days restraint stress (6h daily)

Group 3 (R $10+$ S): Received 21 days stress + resveratrol (10mg/kg body weight dose) for 28 days (Resveratrol was given a week prior to stress treatment)

Group 4 (R $20+$ S): Received 21 days stress + resveratrol ( $20 \mathrm{mg} / \mathrm{kg}$ body weight dose) for 28 days (Resveratrol was given a week prior to stress treatment).
Stressing procedure: Rats were assigned to a restraint stress for 21 days in a wiremesh restrainer for six hours ( $2 \mathrm{hrs} X 3$ times/day). The timings of the stress were different to avoid adaptation to the stress. The wire mesh restrainer had awooden base and stainless steel wire mesh restrainers hinged to the base. The restrainers with dimensions of $11 \mathrm{~cm}(L) \times 8 \mathrm{~cm}$ (B) $x 8 \mathrm{~cm}(\mathrm{H})$ was used to stress. This type of restrainer will only restrict the movements of the animal without causing any pain, discomfort orsuffocation [5].

Resveratrol treatment: Although no studies have yet appeared regarding proper dosage of resveratrol for humans, the optimum beneficiary results were obtained from a dose ranging from 30 to $110 \mathrm{mg} /$ day in human clinical trials [6]. Applying this dose to rat model and also from number of previous studies in rats [7], $10 \mathrm{mg}$ and $20 \mathrm{mg} / \mathrm{kg}$ body weight dose were selected. Resveratrol suspended with $0.5 \%$ carboxy methyl cellulose was administered orally using oropharyngeal tube.

Chemicals: Resveratrol (Cat. No. 70675) was obtained from CaymanChemicals, USA. All other chemicals and reagents were HPLC oranalytical grade (Sigma, St. Louis, Mo.)

Passive avoidance test: To test the memory retention, rat in all the groups were subjected to passive avoidance test as described by Bures et al [8]\&Cherian et al [9]. The test determines the ability of a rat to remember a foot shock delivered $24 \mathrm{~h}$ prior to the memory retention test.

Passive avoidance apparatus consists of a wooden box with two compartments: (a) larger, bright compartment and (b) smaller, dark compartment both equipped with grid floor, which was attached to a shock source. The connection between the two compartments could be closed with a sliding door.

The experiment included three parts, i) an exploration test, ii) an aversive stimulation and learning phase (passive avoidance acquisition), and iii) retention test.

i) Exploration test: On the first day of test, rat was placed in the centre of the illuminated large compartment facing away from the entrance to the dark small compartment, for exploration. The door between the two compartments remained 
open at this time. The rat was allowed to explore both compartments for 5 minutes. This is followed by three test trials of 5 min each. At the end of the trial, the rat was placed in the home cage, where it remained during an interinterval of 5 minutes. In each trial, fraction of time spent in each compartment was noted.

ii) Aversive stimulation and learning phase (passive avoidance acquisition): At the end of 3rd test trial, as soon as the animal stepped into dark compartment, a foot shock was delivered through the grid floor $(50 \mathrm{~Hz}, 1.5 \mathrm{~mA}$, for 1 second). The rat was held additional $10 \mathrm{sec}$, to allow the animal to form an association between the properties of the chamber and foot shock. The rat was then returned to its home cage.

iii) Retention test: The memory retention test was done $24 \mathrm{hr}$ after foot shock. The rat was placed in the bright compartment and the time taken (the step-through latency) for it to enter the dark compartment for the first time was recorded using a stop watch. A maximum of 300 sec were given for the rat to explore. Normal rats avoid entering the dark chamber, where they received shock on previous day, suppressing their normal behaviour of exploring the dark compartment. Decreased latency to enter the dark compartment will suggest poor memory retention.

Antioxidant systems measurement in brain: Tissue processing: Biochemical tests were carried out $24 \mathrm{~h}$ after the last day of stressing. The whole brain was removed rapidly and rinsed with $0.1 \mathrm{M} / \mathrm{L}$ saline phosphate buffer $(\mathrm{pH} 7.4)$. Tissue was weighed and homogenized (1:10w/ v) in $0.1 \mathrm{M} / \mathrm{L}$ saline phosphate buffer. The homogenate was centrifuged at $10,000 \mathrm{X}$ g for $20 \mathrm{~min}$ at $4^{\circ} \mathrm{C}$ and aliquots of supernatant were separated and used for following biochemical estimations.

Assay of reduced glutathione (GSH): Tissue GSH concentration was estimated according to the method described by Ellman [10].

Principle of the assay: The general thiol reagent, 5-5'-dithiobis [2-nitrobenzoic acid] (DTNB, Ellman's Reagent) reacts with GSH to form the $412 \mathrm{~nm}$ chromophore, 5-thionitrobenzoic acid (TNB) and GSH-TNB. The GSH-TNB is subsequently reduced by glutathione reductase and b-nicotinamide adenine dinucleotide phosphate (NADPH), releasing a second TNB molecule and recycling the $\mathrm{GSH}$, thus amplifying the response. Any oxidised (GSSG) initially present in the reaction mixture or formed from the mixed disulphide reaction is rapidly reduced to GSH.

Assay procedure: One millilitre of supernatant was precipitated with $1 \mathrm{ml}$ of metaphosphoric acid and cold digested at $4{ }^{\circ} \mathrm{C}$ for $1 \mathrm{~h}$. The samples were centrifuged at $1,200 \mathrm{~g}$ for $15 \mathrm{~min}$ at 4 으. To $1 \mathrm{ml}$ of this supernatant, $2.7 \mathrm{ml}$ of phosphate buffer and $0.2 \mathrm{ml}$ of 5, 5' dithio-bis2-nitrobenzoic acid (DTNB) was added. The yellow colour that developed was read immediately at $412 \mathrm{~nm}$ using a Systronic-117 UVVisible spectrophotometer. The values were expressed in $\mathrm{mg} / \mathrm{gm}$ protein. The total protein concentration of tissues was measured by the method of Lowry et al [11].

Assay of glutathione reductase (GSH-Rd): The GSH-Rd activity was measured using the method originally described by Moron et al [12].

Principle of the assay: This assay is based on the reduction of oxidized glutathione (GSSG) to reduced glutathione (GSH) by NADPH in the presence of glutathione reductase. The reaction is measured by the decrease in absorbance at $340 \mathrm{~nm}$ using an extinction coefficient of chromospheres $(1.36 \times 104(\mathrm{~mol} / \mathrm{l})-1 \mathrm{~cm}-1)$. The activity of glutathione reductase was used as an indicator for oxidative stress.

Assay procedure: The reaction mixture consisted of $1.6 \mathrm{ml}$ of $0.067 \mathrm{M}$ potassium phosphate buffer (pH 6.6), $0.12 \mathrm{ml}$ of $0.06 \%$ $\mathrm{NADPH}, 0.12 \mathrm{ml} 1.15 \% \mathrm{GSSG}, 0.1 \mathrm{ml}$ of enzyme source and water in a final volume of $2 \mathrm{ml}$. All mixtures and solutions were prepared at room temperature. Control cuvettes then received $180 \mu \mathrm{L}$ of deionized water while sample cuvettes received $60 \mu \mathrm{L}$ of deionized water and $120 \mu \mathrm{L}$ of GSSG solution. NADPH oxidation was followed for $5 \mathrm{~min}$ and was recorded using a Systronic117 spectrophotometer. The reduction of GSSG to GSH was determined indirectly by the measurement of the consumption of NADPH, as demonstrated by a decrease in absorbance at $340 \mathrm{~nm}$ as a function of time. The enzyme activity was calculated using extinction coefficient of 
chromophore $(1.36 \times 104(\mathrm{~mol} / \mathrm{l})-1 \mathrm{~cm}-1)$ and expressed as nmol NADPH oxidized/min/mg protein. Protein content was determined by the method of Lowry et al [11] with bovine serum albumin as standard.

Histological study of Dentategyrus, hippocampus and medial pre-frontal cortex: Each rat was deeply anesthetized with ether and secured on a dissection board, and its chest cavity wasopened to expose the heart. About $15 \mathrm{~mL}$ of $0.9 \%$ saline was perfusedthrough the left ventricle at the rate of $1 \mathrm{~mL} / \mathrm{min}$. This was followedby perfusion of $10 \%$ formalin at the same flow rate. The animal wasdecapitated and the brain was removed and kept in $10 \%$ formalinfor $48 \mathrm{~h}$ (post-fixation). Paraffin blocks were made in an embeddingbath. Coronal sections of 5-ìm thickness were cut in the dorsalhippocampus using a rotary microtome (Jung Biocutt 2035, Leica,Germany). Ten sections from each rat were mountedserially on air-dried gelatinized slides.

The sections were stained with cresyl violet stain. One hundred milligramsof cresyl violet was dissolved in $100 \mathrm{~mL}$ of distilled water. Tothis, $0.5 \mathrm{~mL}$ of $10 \%$ acetic acid was added to give a $\mathrm{pH}$ of 3.5-3.8. Thestain was filtered before use [13]

Scoring: Quantification was done using Nikon trinocular microscope (H600L) under 40X magnificationusing imaging software NIS Elements $\mathrm{Br}$ version 4.30. For various regions of the hippocampus $250 \mu \mathrm{m}$ length area was selected, for dentate gyrus $240 \mu \mathrm{m}^{2}$ area and for medial prefrontal cortex $300{\mu \mathrm{m}^{2}}^{2}$ area was selected for quantification of normal neurons. Slides from different groups of rats were decoded to avoid manualbias while counting the cells.

Statistical Analysis: The data were expressed as mean \pm SD. The significance ofdifferences among the groups were assessed using one way analysisof Variance (ANOVA) test followed by Bonferroni's multiplecomparison test. $P$ values< 0.05 were considered as significant.

\section{RESULTS}

The general health conditions of the rats during stressing/treatment period were monitored carefully; body weights were noted at frequent interval to ensure that no other ill health affects the results. No such effects were observed among all the rats tested.

Passive avoidance test: Rats who received restraint stress took significantly $(p<0.001)$ lesser time to enter the dark compartment when compared to control. This shorter latency indicates poor retrieval of learning behaviour. Resveratrol treatment in stressed rat did not show any increase in time interval to enter the dark compartment compared to control as the latency to enter the dark compartment were significantly less $(p<0.001)$ when compared to control. This would indicate a poor retrieval of learning behaviour in stressed rats even after resveratrol treatment. However, resveratrol at both doses has enhanced latency to enter the dark compartment in stressed rats compared to rats who received only stress(Fig.1).

\section{Reduced glutathione (GSH) level in brain:} There is a significant $(p<0.001)$ reduction in reduced glutathione level (GSH) in stressed rat brain homogenate compared to the control.Resveratrol treatment (both doses) in stressed rats reversed the oxidative damage by elevating reduced glutathione level significantly $(p<0.001)$. Resveratrol treatment at $10 \mathrm{mg} / \mathrm{kg}$ dose in stressed rats has shown an increase in the reduced glutathione level at par with control (as there is no statistical significant difference between their values),(Fig.2).

Fig. 1: Observations of passive avoidance test by rats subjected to restraint stress and resveratrol treatment.

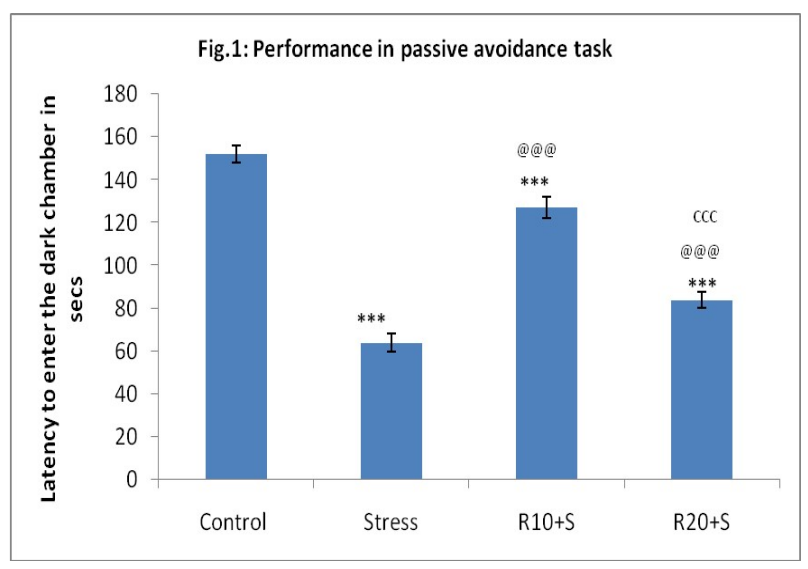

Values are expressed as mean \pm SD $(n=6)$. Comparison between Control Vs others $* *=p<0.01, * * *=p<0.001$. Comparison between Stress Vs others @@@ $0<<0.001$. Comparison between R10+SVs R20 +S CCC $=p<0.001$ 
Fig.2: Brain reduced Glutathione level (mg/gm protein) in rats.

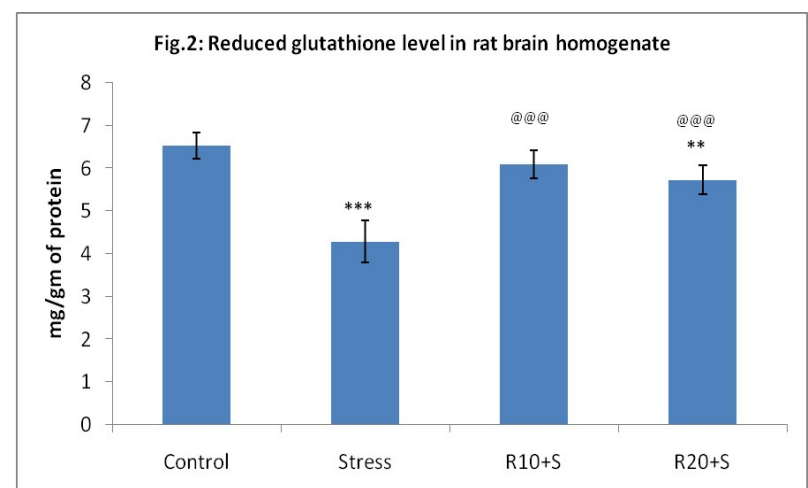

Values are expressed as mean $\pm S D(n=6)$. Comparison between Control Vs others $* *=p<0.01, * * *=p<0.001$. Comparison between Stress Vs others @@@= $0<0.001$.

Glutathione reductase (GSSG-Rd) activity in brain: The activity of brain glutathione reductase enzyme was significantly $(p<0.001)$ decreased in stressed rats compared to control. Resveratrol treatment (both doses) in stressed rats has significantly $(p<0.001)$ increased glutathione reductase activity compared to rats who received only stress claiming the antioxidant potential of resveratrol in stressed conditions. Resveratrol treatment at $10 \mathrm{mg} / \mathrm{kg}$ dose in stressed rats has shown an increase in the glutathione reductase level at par with control (as there is no statistical significant difference between their values), (Fig.3).

Fig.3: Brain Glutathione reductase activity (nmol NADPH oxidized/min/mg protein) in rats.

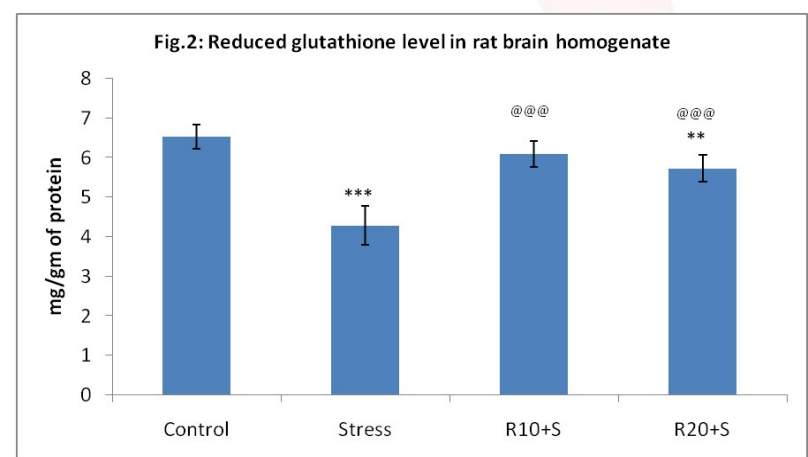

Values are expressed as mean $\pm \operatorname{SD}(n=6)$. Comparison between Control Vs others $* * *=p<0.001$. Comparison between Stress Vs others @@@=p<0.001. Comparison between R10+SVs R20+S CCC $=p<0.001$.

\section{Glutathione reductase (GSSG-Rd) activity in} brain: The activity of brain glutathione reductase enzyme was significantly $(p<0.001)$ decreased in stressed rats compared to control. Resveratrol treatment (both doses) in stressed rats has significantly $(p<0.001)$ increased glutathione reductase activity compared to rats who received only stress claiming the antioxidant potential of resveratrol in stressed conditions. Resveratrol treatment at $10 \mathrm{mg} / \mathrm{kg}$ dose in stressed rats has shown an increase in the glutathione reductase level at par with control (as there is no statistical significant difference between their values), (Fig.3).

Fig.3: Brain Glutathione reductase activity (nmol NADPH oxidized/min/mg protein) in rats.

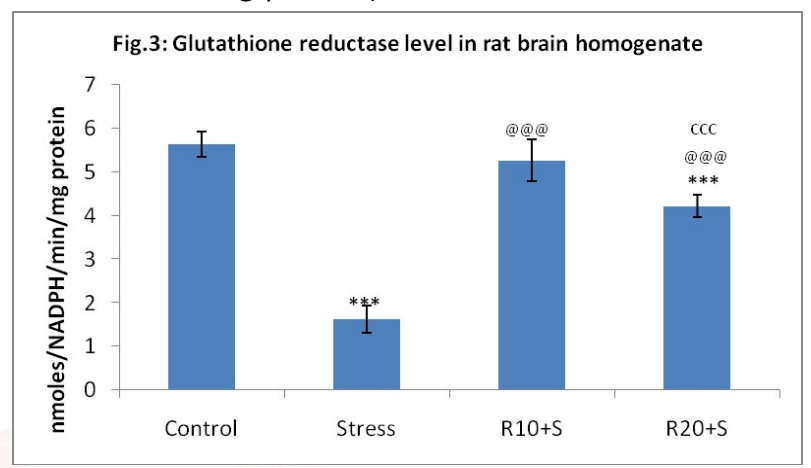

Values are expressed as mean \pm SD $(n=6)$. Comparison between Control Vs others $* * *=p<0.001$. Comparison between Stress Vs others @@@= p<0.001. Comparison between R10+SVs R20+S CCC $=p<0.001$.

Neuronal assay of Dentate Gyrus (DG): The expression of number of healthy neurons in DG has reduced significantly $(p<0.001)$ in stressed rats compared to control indicating a neuronal loss. The number of neurons were significantly $(p<0.001)$ more in stressed rats who received resveratrol compared to the group which received stress alone. Rats who received stress and resveratrol $10 \mathrm{mg} / \mathrm{kg}$ dose expressed higher $(p<0.01)$ number of neurons compared to rats who received stress and $20 \mathrm{mg} / \mathrm{kg}$ dose of resveratrol (Fig.4,5a \& 5b) suggesting $10 \mathrm{mg} / \mathrm{kg}$ dose acting better.

Fig. 4: Resveratrol and their combination induced changes in the neuronal numbers in 240 squm 2 area in Dentate Gyrus (DG).

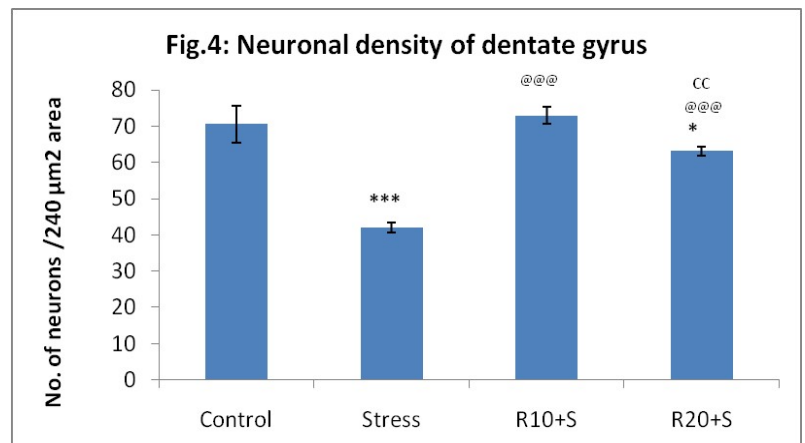

Values are expressed as mean $\pm S D(n=24$ slides/group). Comparison between Control Vs others $* * *=p<0.001, *$ $=p<0.05$. Comparison between Stress Vs others @@@ = $p<0.001$. Comparison between R10+SVs R20 +S CC $=p<0.01$. 
Fig. 5: Histomicrographic pictures of dentate gyrus under 40X, Cresyl violet staining. Note degenerated neurons indicated by red arrow in stressed group of rats.

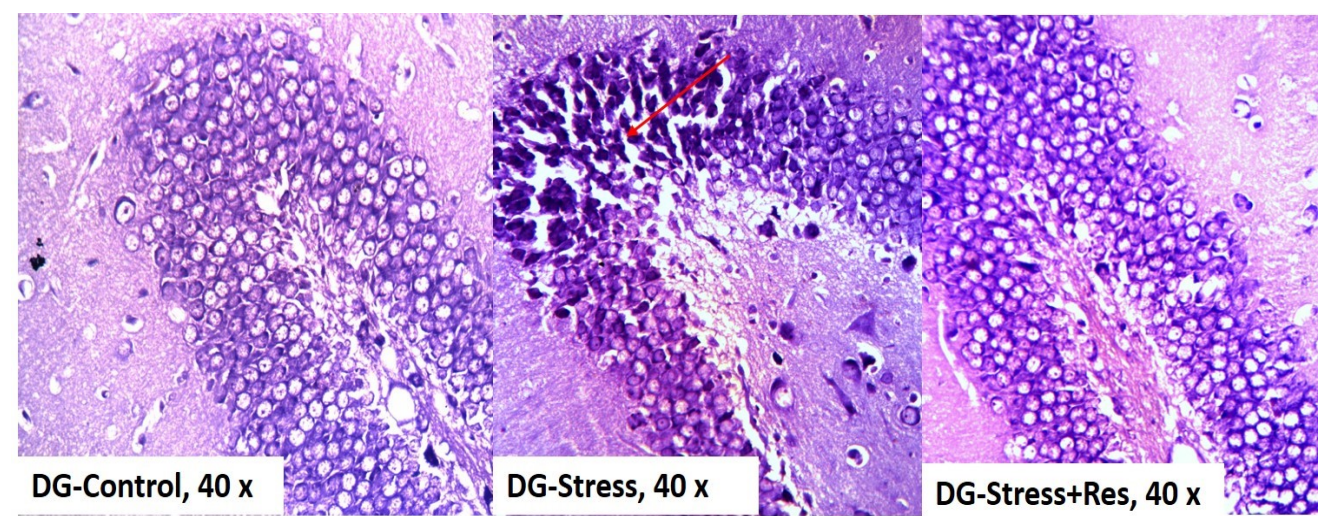

Fig. 6: Resveratrol and their combination induced changes in the neuronal numbers in $250 \mu \mathrm{m}$ length area in hippocampal CornuAmmonis (CA4, CA3, CA2, CA1).

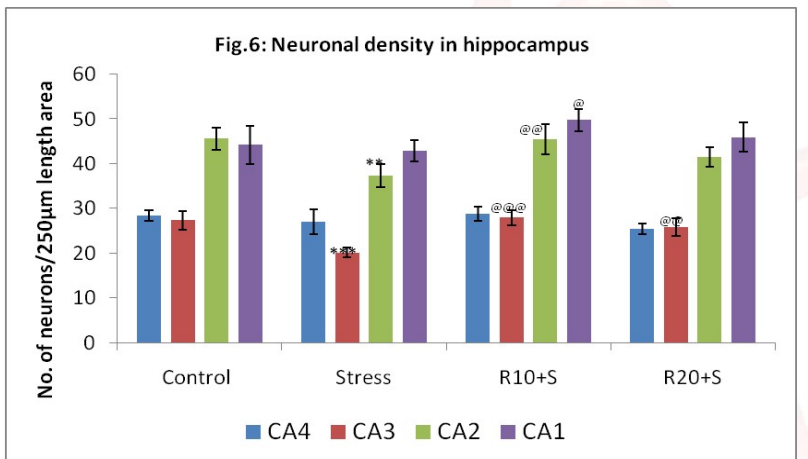

Values are expressed as mean \pm SD $(n=6)$. Comparison between Control Vs others $* *=p<0.01, * * *=p<0.001$. Comparison between Stress Vs others @@=p<0.01, @@@=p<0.001.

Fig. 7: Histo micrographic pictures of hippocampus under 40X, Cresyl violet staining. Scale bar indicates $50 \mu \mathrm{m}$ length.

Fig. 9: Histo micrographic pictures of medial prefrontal cortex (MFC) under 40X,

Cresyl violet staining.
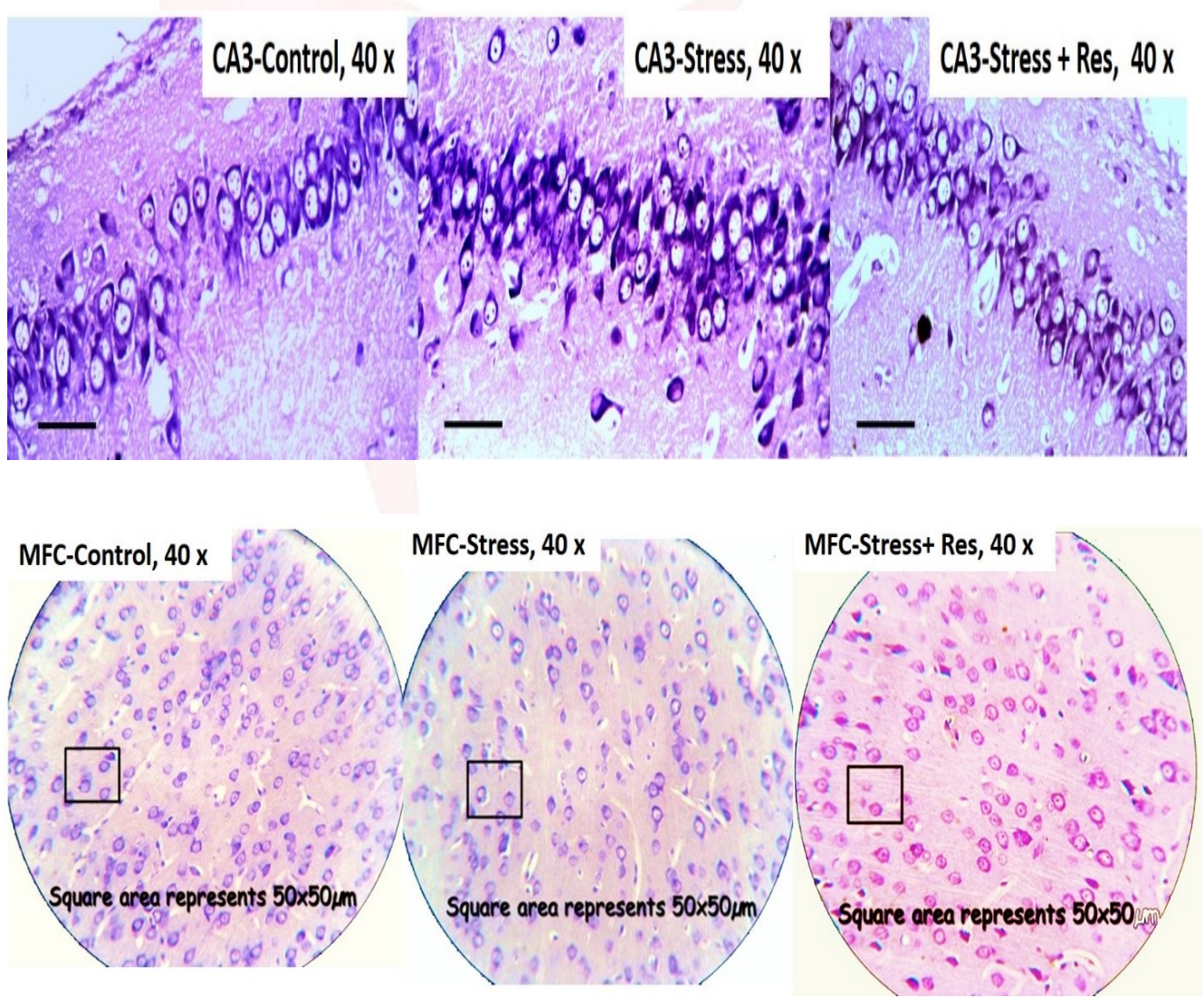
Neuronal assay of various regions of the Hippocampus: Restraint stress has not affected ( $p>0.05$ ) the neuronal population of CA4 and CA1 regions of the hippocampus. However CA3 region showed a severe $(p<0.001)$ decline in neuronal number followed by CA2 region $(p<0.01)$ in stressed rats compared to control counterparts.

Resveratrol at $10 \mathrm{mg} / \mathrm{kg}$ dose in stressed rats enhanced $(p<0.001)$ the expression of normal neurons in $\mathrm{CA} 3$ region and also in CA2 region to a lesser $(p<0.01)$ extent. Resveratrol at $20 \mathrm{mg} /$ $\mathrm{kg}$ dose in stressed rats also enhanced $(p<0.01)$ the expression of normal neurons in CA3 region but not ( $p>0.05$ ) at CA2 region (Fig.6, 7a \& 7b).

Neuronal assay of medial prefrontal cortex: Restraint stress has expressed significantly $(p<0.001)$ lesser number of neurons in stressed rats compared to control. Resveratrol at both doses in stressed rats showed a significant $(p<$ 0.001 ) higher number of neurons compared to rats who received stress alone (Fig.8, 9a \& 9b).

\section{DISCUSSION}

The results of the present study revealed poor retrieval of learning behaviour in passive avoidance task after restraint stress. Though the restraint stress-induced cognitive dysfunction was reported in many animal studies most of them assessed hippocampal dependent spatial memory using variety of maze tests. The present study was designed to evaluate the cognitive function in an unconditioned learning environment and our results further demonstrates the involvement of hippocampal as well as prefrontal cortex neurons involving in it. Chronic restraint stress induced cognitive dysfunction involving hippocampus [14] and prefrontal cortex [15],[16] in humans and animal models were well established [17]. There are number of hypotheses addressing the mechanism of stress induced cognitive dysfunction. It includes oxidative damage, altered glucocorticoid receptor expression [18], altered neurotransmitter and synaptic proteins [19], neuronal $\mathrm{Ca} 2+$ homeostatis disturbance [20], altered dendritic morphology [21] and many more. Among the various factors affecting the stress-induced cognitive dysfunction, the involvement of in built antioxidant defence system in brain is gaining lot of focus in the recent years. It is because of the fact, that in age related dementia and in Alzheimer's disease the neuronal loss was mainly due to loss of antioxidant defence system [22] in the brain specially in hippocampus [23] and prefrontal cortex [24]. For these reason natural antioxidants crossing blood brain barrier like resveratrol was used as therapeutic strategy. Resveratrol has exerted its antioxidant potential in enhancing cognitive function against various neuronal insults[25],[26],[27],[28],[29],[30]. In the present study though resveratrol has not enhanced retrieval of learning behaviour to the normalcy, but it was better than the performance of stressed rats. Number of studies in the past using different study models have demonstrated resveratrol's ability to prevent cognitive loss. The mild difference observed in the results of various study models, may be due to sensitivity of rats for each study design. In the present study the neuroprotective role of resveratrol against restraint stress-induced cognitive dysfunction involving brain glutathione system is further elaborated.

Reactive oxygen species (ROS) generated by chronic restraint stress significantly hampers the in-built antioxidant system in rat brain [1].In the present study chronic restraint stress has caused a decline in reduced glutathione level in rat brain homogenate and this effect was reversed by resveratrol treatment. Glutathione (GSH) exerts an important function in defence against membrane peroxidation and also by reducing hydrogen peroxide with glutathione peroxidase. In cells GSH is retained in their reduced form by the glutathione reductase and in turn reduces other metabolites and enzyme systems as well as reacting directly with oxidants. Reduced glutathione is essential for the cellular detoxification of ROS in brain cells [31]. Immobilization stress causing a decrease in the brain levels of glutathione [32] was reported. In this study resveratrol has significantly elevated the brain glutathione level in stressed rats. Similar protective effect of resveratrol was observed after traumatic brain injury by expressing elevated reduced glutathione level [33]. The study by $\mathrm{Ma}$ et al[25] and Tiwari \& Chopra[26], also show elevated brain glutathione level after resveratrol treatment against vascular dementia and 
alcohol induced toxicity models respectively. Now this study further confirms antioxidant role against stress-induced free-radical generation.

The function of glutathione reductase enzyme is to produce reduced glutathione from oxidised glutathione, maintaining high ratio of reduce to oxidised form intracellularly. In the present study chronic restraint stress expressed a severe decline in this enzyme level. Resveratrol at $10 \mathrm{mg} /$ $\mathrm{kg}$ dose has protected this oxidative damage by bringing the glutathione reductase level to the normalcy. Resveratrol has exerted its antioxidant potential in obese mice associated with increase in cerebral oxidative stress [34].

Elevation in reduced glutathione and glutathione reductase enzyme observed in this study can be attributed to free radical scavenging properties of resveratrol. Being a well-known antioxidant resveratrol could inhibit free radical generation in brain and spinal cord [35],[36]. It is known to hinder the lipid peroxidation [13] and inhibits apoptotic cell death produced by oxidative stress [37]. It has been well claimed that resveratrol could inhibit mitochondria-induced production of ROS in rat brain [38], protect DNA from oxidative damage in stroke-prone hypertensive rats [39] and could prevent neuronal loss after ischemia/reperfusion injury.

The impact of oxidative stress to nervous tissue is numerous, as nervous tissue is particularly vulnerable to oxidative stress due to its high rate of oxygen consumption. Stress-induced oxidative damage involves mitochondrial dysfunction, dysregulation of $\mathrm{Ca} 2+$ homeostatis[40], damage to neuronal stem cells (defective neurogenesis) [41], induction of signaling events in apoptotic cell death [42]. Oxidative stress eventually leads to morphological changes and finally neuronal atrophy/death [43]. Hence in the present study we further focussed on neuronal morphology of the areas concerned with cognition.

The present study reveals a selective and quantitative neuronal loss affecting DG, CA3, CA2 and MPFC. Restraint stress induced neuronal loss in animal models is an established fact. Each neuronal population has a unique molecular composition that determines its level of vulnerability to oxidative stress. Results of the study by Tong et al[44] on animal model conclude that oxidative stress due to traumatic brain injury specifically involves CA2, CA3 regions of the hippocampus and DG. The region specific loss of neurons after restraint stress is known to involve many factors of which high density of corticosteroid receptors in DG and CA3 region which are vulnerable to glucocorticoids released due to stress. This would cause inhibition of glucose transport and elevation of cytoplasmic calcium concentration. The loss of neurons observed is consistent with breakdown of brain glutathione system in the rat brain. DG is an area where active neurogenesis continues throughout the adulthood in humans as well as animals. Functionally the projection of axons of granule cells of DG into CA3 region of hippocampus is a primary circuit for spatial memory. The turnover of granule cells of the DG in adult life is required for hippocampal function in spatial memory [45]. In our study restraint stress has shown neuronal loss in the dentate gyrus. Resveratrol in stressed rats has minimized this cytotoxic effect.

Long lasting stress inhibits proliferation and survival capacity of newly born neuronal stem cells [46]. The neuronal stem cells arising from DG differentiates and begin to migrate to the granule cell layer. This neurogenesis and survival are regulated negatively by glucocorticoids (as in stress), excitatory amino acids and opioids. Defective neurogenesis [47] or loss of adult neurons [48] is always associated with decline in learning abilities as well as memory retention as observed in the present study.

Medial prefrontal cortex (MPFC) is involved in both memory and decision making. But its functions like learning and memory consolidation depends on its connection with hippocampus [49]. Quinn et al[50] concluded that MPFC is necessary for both recent and remote memory. Both MPFC and hippocampus is necessary for consolidation of memory after learning. It is also known that MFC is involved in retrieval of memory after a task for subsequent days [49]. In the present study model the foot shock escaping task were continued for 5 days, observing the animal's ability to retrieve the memory during 5 days and also after a week. Loss of neurons in the MPFC with declined retrieval capability was observed after chronic stress which was reversed by resveratrol. From the earlier 
studies the interaction between hippocampus and MPFC is necessary for consolidation of memory after learning. Quantitative loss of neurons in both the regions can be attributed to cognitive decline.

\section{CONCLUSION}

To conclude, the chronic restraint stress induced cognitive decline in the present study can be attributed to break down of brain glutathione system and region specific neuronal loss. However estimation of reduced glutathione and glutathione reductase and other specific enzymes in hippocampal and frontal cortex region would have thrown more light on this observation. In this study resveratrol at all the three (behavioural, biochemical and morphological) levels showed beneficial effect. Hence in clinically diagnosed condition of stress, resveratrol would be a therapeutic option, as stress is integral part of current day's life style.

\section{Conflicts of Interests: None \\ REFERENCES}

[1]. Zaidi SM, Al-Qirim TM, Banu N. Effects of antioxidant vitamins on glutathione depletion and lipid peroxidation induced by restraint stress in the rat liver. Drugs R D 2005; 6(3):157-165.

[2]. Qi-Hai Gong, Fe Li, Feng Jin, Jing-Shan Shi. Resveratrol attenuates neuroinflammation-mediated cognitive deficits in rats, J Health Sci 2010; 56:655-663.

[3]. Blanchet J, Longpre F, Bureau G, Morissette M, DiPaolo T, Bronchti $\mathrm{G}$ et al. Resveratrol, a red wine polyphenol, protects dopaminergic neurons in MPTP-treated

mice. ProgNeuropsychopharmacolBiol Psychiatry 2008; 32:1243-1250.

[4]. Jin F, Wu Q, Lu YF, Gong QH, Shi JS. Neuroprotective effect of resveratrol on 6-OHDA-induced Parkinson's disease in rats. Eur J Pharmacol 2009; 600:78-82.

[5]. Madhyastha S, Prabhu LV, Nayak SR, Pai M, Rajalakshmi, Madhyastha P. Effect of prenatal stress and serotonin depletion on postnatal serotonin metabolism in wistar rats. Iran J PharmacolTher 2008; 7:71-77.

[6]. Bone K. Studies Provide Insights into Effective Clinical Dose of Resveratrol. Medi herb 2010; e-Monitor 34.

[7]. Kumar A, Naidu PS, Seghal N, Padi S S. Neuroprotective effects of resveratrol against intra cerebro-ventricular colchicine induced cognitive impairment and oxidative stress in rats. Pharmacology 2007; 79:17-26.

[8]. Bures J, Buresova O, Huston JP. Techniques and basic experiments for the study of brain and behavior. 2nd edition: Elsevier Science Publishers, Amsterdam/Newyork. 1983; 148-160.
[9]. Cherian SB, Bairy KL, Rao MS. Chronic prenatal restraint stress induced memory impairment in passive avoidance task in post weaned male and female Wistar rats. Ind J ExptBiol 2009; 47; 893-899.

[10].Ellman GL. Tissue sulfhydryl groups. Arch BiochemBiophys 1959; 82:70-77.

[11]. Lowry OH, Rosebrough NJ, Farr AL, Randall AJ. Protein measurement with the Folin phenol reagent. J Biol Chem. 1951; 193(1):265-275.

[12]. Moron MS, Depierre JW, Mannervik B. Levels of glutathione, glutathione reductase and glutathione $S$ transferease activities in rat lung and liver. BiochemBiophysActa 1979; 582:67-78.

[13]. Madhyastha S, Sahu SS, Rao Gayathri. Resveratrol for prenatal-stress-induced oxidative damage in growing brain and its consequences on survival of neurons. J Basic ClinPhysiolPharmacol 2014: 25(1):63-72.

[14]. Bodnoff SR, Humphrey AG, Lehman JC, Diamond DM, Rose GM, Meaneyl MJ. Enduring Effects of Chronic Corticosterone Treatment on Spatial Learning, Synaptic Plasticity, and Hippocampal Neuropathology in Young and Mid-Aged Rats. The Journal of Neuroscience 1995; 15(1):61-69.

[15]. McEwen BS. Physiology and neurobiology of stress and adaptation: central role of the brain. Physiol Rev. 2007; 87(3):873-904.

[16]. Radley JJ, Rocher AB, Miller M, Janssen WGM, Liston C, Hof PR et al., Repeated Stress Induces Dendritic Spine Loss in the Rat Medial Prefrontal Cortex. Cerebral Cortex 2006; 16:313-320.

[17]. Caso JR, Pradillo JM, Hurtado O, Leza JC, Moro M, Lizasoain I. Toll-like receptor 4 is involved in subacute stress-induced neuroinflammation and in the worsening of experimental stroke. Stroke; a journal of cerebral circulation 2008; 39(4):1314-1320.

[18]. Lupien SJ, McEwen BS. The acute effects of corticosteroids on cognition: integration of animal and human model studies. Brain Res. Rev. 1997; 24:1-27.

[19]. Khurana RC, Devaud LL. Sex differences in neurotransmission parameters in response to repeated mild restraint stress exposures in intact male, female and ovariectomised female rats. Journal of neuroendocrinology 2007; 19(7):511-520.

[20]. Augustine GJ, Santamaria F, Tanaka K. Local calcium signalling in neurons. Neuron 2003; 40:331346.

[21]. Manikandan S, Padma MK, Srikumar R, JeyaParthasarathy $N$, Muthuvel A, Sheela Devi R. Effects of chronic noise stress on spatial memory of rats in relation to neuronal dendritic alteration and free radical-imbalance in hippocampus and medial prefrontal cortex. Neuroscilett 2006; 15:399(1-2):17-22.

[22]. Eghwrudjakpor PO, Allison AB. Oxidative stress following traumatic brain injury: enhancement of endogenous antioxidant defense systems and the promise of improved outcome. Niger J Med. 2010; 19(1):14-21.

[23]. Padurariu M, Ciobica A, Mavroudis I, Fotiou D, Baloyannis S Hippocampal Neuronal Loss In The 
T. Kalpana et al. RESVERATROL REVERSES BRAIN GLUTATHIONE SYSTEM INVOLVED NEURONAL LOSS AFTER IMMOBILIZATION STRESS

Ca1 And Ca3 Areas Of Alzheimer's Disease Patients. PsychiatriaDanubina 2012; 24(2):152-158.

[24]. Ansari MA, Scheff SW. Oxidative Stress in the Progression of Alzheimer Disease in the Frontal Cortex. J NeuropatholExpNeurol 2010; 69(2):155-167.

[25]. Ma X, Sun Z, Liu Y, Jia Y, Zhang B, Zhang J. Resveratrol improves cognition and reduces oxidative stress in rats with vascular dementia. Neural regeneration research 2013; 8(22):2050-2059.

[26] Tiwari V, Chopra K. Resveratrol prevents alcohol induced cognitive deficits and brain damage by blocking inflammatory signalling and cell death cascade in neonatal rat brain. Journal of neurochemistry 2011; 117:678-690.

[27]. Pushpalatha B, Venumadhav N, Swathi M, Raju AB. Neuroprotective effect of resveratrol against scopolamine-induced Cognitive impairment and oxidative stress in rats. Arch. Biol. Sci., Belgrade 2013; 65(4):1381-1386.

[28].Meng XJ, Wang F, Li CK. Resveratrol is Neuroprotective and Improves Cognition in Pentylenetetrazole kindling Model of Epilepsy in Rats. Indian Journal of Pharmaceutical Sciences 2014; 76(2):125-131.

[29]. Pilsakova L, Riecansky I, Jagla F. The physiological actions of isoflavone phytoestrogens. Physiol Res 2010; 59:651-664.

[30]. Madhyastha S, Sahu SS, Rao Gayathri. Prenatal stress-induced cognitive impairment and neuronal oxidative stress and its amelioration by resveratrol in neonate rats. Res J Of Pharmaceutical, Biological and Chemical Sciences 2012:3(4); 1387-1399.

[31]. Dringen R, Hirrlinger J. Glutathione pathways in the brain. J BiolChem 2003; 384:505-516.

[32]. Zaidi SK, Md. Nasrul H, Tabrez S, Ansari SA, Jafri MA, Khan MS et al. Protective Effect of Solanumnigrum Leaves Extract on Immobilization Stress Induced Changes in Rat's Brain. Evidence-Based Complementary and Alternative Medicine 2014; Article ID 912450:1-7.

[33]. Ates O, Cayli S, Altinoz E, Gurses I, Yucel N, Sener M et al. Neuroprotection by resveratrol against traumatic brain injury in rats. Molecular and Cellular Biochemistry 2007; 294:137-144.

[34]. Rege SD, Kumar S, Wilson DN, Tamura L, Geetha T, Mathews ST et al., Resveratrol Protects the Brain of Obese Mice from Oxidative Damage. Oxidative Medicine and Cellular Longevity 2013; Article ID 419092:1-7.

[35]. Yang YB, Piao YJ. Effects of resveratrol on secondary damages after acute spinal cord injury in rats. ActaPharmacol Sin 2003; 24:703-710.

[36]. Inoue H, Jiang XF, Katayama T, Osada S, Umesono K, Namura S. Brain protection by resveratrol and fenofibrate against stroke requires peroxisome proliferator-activated receptor a in mice. Neuroscilett 2003; 352:203-206.

[37]. Chanvitayapongs S, Draczynska-Lusiak B, Sun AY. Amelioration of oxidative stress by antioxidants and resveratrol in PC12 cells. Neuroreport 1997; 8:1499-1502.
[38]. Zini R, Morin C, Berteli A, Bertelli AA, Tillement JP. Effects of resveratrol on the rat brain respiratory chain. Drugs ExpClin Res 1999; 25:87-97.

[39]. Mizutani K, Ikeda K, Kawai Y, Yamori Y. Protective effective of resveratrol on oxidative damage in male and female stroke-prone spontaneously hypertensive rats. ClinExpPharmacolPhysiol 2001; 28:5559.

[40]. Amoroso S, D’Alessio A, Sirabella R, Di Renzo G, Annunziato $L$. Ca2+-independent caspase-3 but not Ca2+-dependent caspase- 2 activation induced by oxidative stress leads to $\mathrm{SH}-\mathrm{SY} 5 \mathrm{Y}$ human neuroblastoma cell apoptosis. J Neurosci Res. 2000; 68:45462.

[41]. Kroemer G. The proto-oncogene $\mathrm{Bcl}-2$ and its role in regulating apoptosis. Nat Med 1997; 3: 614-620.

[42]. Cregan SP, Fortin A, MacLaurin JG, Callaghan SM, Cecconi F, Yu SW. Apoptosis-inducing factor is involved in the regulation of caspase-independent neuronal cell death. J Cell Biol 2002; 158:507-17.

[43]. Sapolsky RM. The possibility of neurotoxicity in the hippocampus in major depression: a primer on neuronal death. Biol Psychiatry 2000; 48:755-765.

[44]. Tong W, Igarashi T, Ferriero DM, Noble LJ. Traumatic brain injury in the immature mouse brain: characterization of regional vulnerability ExpNeurol 2002; 176:105-116.

[45]. Sherry DF, Jacobs LF, Gaulin SJ. Spatial memory and adaptive specialization of the hippocampus, Trends Neurosci 1992; 15:298-303.

[46]. Torner L, Karg S, Blume A, Kandasamy M, Kuhn HG, Winkler J et al. Prolactin prevents chronic stressinduced decrease of adult hippocampal neurogenesis and promotes neuronal fate. J Neurosci 2009; 29:1826-1833.

[47]. Lagace DC, Donovan MH, DeCarolis NA, Farnbauch LA, Malhotra S, Berton O et al. Adult hippocampal neurogenesis is functionally important for stress induced social avoidance. ProcNatlAcadSci 2010; 107(9):4436-4441.

[48].Sapolsky R, Krey L, McEwen BS. Prolonged glucocortiocoid exposure reduces hippocampal neuron number: Implications for aging. J Neurosci 1985; 5:1121-1127.

[49]. Euston DR, Gruber AJ, McNaughton BL. The Role of Medial Prefrontal Cortex in Memory and Decision Making. Neuron 2012; 76:1057-1070.

[50]. Quinn JJ, Ma QD, Tinsley MR, Koch C, Fanselow MS. Inverse temporal contributions of the dorsal hippocampus and medial prefrontal cortex to the expression of long-term fear memories. Learn. Mem 2008; 15:368-372.

How to cite this article: T. Kalpana, Ramya Sree A, B. Naveen Kumar. RESVERATROL REVERSES BRAIN GLUTATHIONE SYSTEM INVOLVED NEURONAL LOSS AFTER IMMOBILIZATION STRESS. Int J Anat Res 2019;7(3.1): 6691-6700. DOI: 10.16965/ijar.2019.160 